\title{
Multimodal outcome assessment after surgery for brainstem cavernous malformations
}

\author{
Philipp Dammann, MD, ${ }^{1}$ Annika Herten, MD, ${ }^{1}$ Alejandro N. Santos, MD, ${ }^{1}$ \\ Laurèl Rauschenbach, MD, ${ }^{1}$ Bixia Chen, MD, ${ }^{1}$ Marvin Darkwah Oppong, MD, ${ }^{1}$ \\ Börge Schmidt, PhD, ${ }^{2}$ Michael Forsting, MD, ${ }^{3}$ Christoph Kleinschnitz, MD, ${ }^{4}$ and Ulrich Sure, MD ${ }^{1}$
}

\begin{abstract}
${ }^{1}$ Department of Neurosurgery, University Hospital Essen; ${ }^{2}$ Institute for Medical Informatics, Biometry and Epidemiology, University Hospital Essen; ${ }^{3}$ nstitute of Diagnostic and Interventional Radiology and Neuroradiology, University Hospital Essen; and ${ }^{4}$ Department of Neurology, University Hospital Essen, University of Duisburg-Essen, Essen, Germany
\end{abstract}

OBJECTIVE The object of this study was to assess outcome after surgery for brainstem cavernous malformations (BSCMs) using functional, health-related quality of life (HRQOL), and psychological surveys to analyze the interrelation of these measurements, and to compare HRQOL and anxiety and depression scores with those in a healthy population.

METHODS The authors performed a cross-sectional outcome study of all patients surgically treated for BSCM in their department between January 1, 2003, and December 31, 2019. They assessed functional outcome via the modified Rankin Scale (mRS), health-related quality of life (HRQOL) via the SF-36 and 9-item Life Satisfaction Questionnaire (LISAT-9), cranial nerve and brainstem function using a questionnaire, symptom-based psychological outcome via the Hospital Anxiety and Depression Scale (HADS), and timepoint of a return to previous employment. They analyzed the correlation between absolute ( $\mathrm{mRS}$ score $\leq 2$ ) and relative (postoperative deterioration in initial $\mathrm{mRS}$ score) outcome endpoints and the interrelation of the outcome measures and performed a comparison of HRQOL and HADS scores with findings in a healthy population.

RESULTS Seventy-four patients were eligible for inclusion in the study. HRQOL was impaired after surgery for BSCM compared to that in a healthy population. This impairment was substantial in patients with an unfavorable functional outcome (mRS > 2) but was also present in those with a favorable outcome (mRS $\leq 2)$ in selected domains. Psychological impairment was negligible in patients with a favorable outcome and grave in those with an unfavorable outcome. LISAT-9 results revealed that brainstem and cranial nerve symptoms reduce satisfaction mainly in self-care abilities for both unfavorable and favorable outcome patients. Among the brainstem and cranial nerve symptoms, balance impairment showed the most significant impact on HRQOL. Absolute outcome endpoints were superior to relative outcome endpoints in reflecting impairment in HRQOL after surgery.

CONCLUSIONS The study data can improve patient counseling and decision-making in BSCM treatment and may function as a benchmark. The authors report outcomes after BSCM surgery in high detail, emphasizing the specific impact of cranial nerve and brainstem symptoms on HRQOL. When reporting BSCM surgery outcome, absolute outcome endpoints should be applied.

https://thejns.org/doi/abs/10.3171/2020.6.JNS201823

KEYWORDS brainstem cavernous malformation; surgery; health-related quality of life; functional outcome; CCM; cerebral cavernous malformation; cavernous angioma; HRQOL; vascular disorders

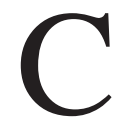
EREBRAL cavernous malformations (CCMs) are prone to intracerebral hemorrhage. ${ }^{1,2}$ And while hemorrhages from CCMs are oftentimes mild and can even remain unrecognized by the patient,${ }^{3-5}$ symptomatic hemorrhages (term defined in reporting standards) ${ }^{6}$ occur with an overall annual risk of $2 \%-6 \%$ and can cause seizures or focal neurological deficits. 7,8 Especially when located in the brainstem, hemorrhage from CCM is more often symptomatic and causes more severe disability than that due to supratentorial CCM. ${ }^{1,8-11}$ Brainstem CM (BSCM) has been found in roughly $30 \%$ of the cases in larger series. ${ }^{1}$ The 5 -year risk of a recurrent hemorrhage

ABBREVIATIONS AUC $=$ area under the curve; $B S C M=$ brainstem cavernous malformation; $C C M=$ cerebral cavernous malformation; HADS $=$ Hospital Anxiety and Depression Scale; HADS-A = Anxiety Subscale of HADS; HADS-D = Depression Subscale of HADS; HRQOL = health-related quality of life; LISAT-9 = 9-item Life Satisfaction Questionnaire; MCS = mental component summary of SF-36; $m R S=$ modified Rankin Scale; PCS = physical component summary of SF-36; ROC = receiver operating characteristic.

SUBMITTED May 15, 2020. ACCEPTED June 9, 2020.

INCLUDE WHEN CITING Published online October 16, 2020; DOI: 10.3171/2020.6.JNS201823. 
in $\mathrm{BSCM}$ is $30.8 \%$ compared to $18.4 \%$ in nonbrainstem CCM. ${ }^{1}$ The potential to cause permanent neurological damage by (repetitive) hemorrhages makes patients with BSCM candidates for neurosurgical resection of the lesion. ${ }^{12}$ Surgical treatment rates of BSCM range around $20 \%-25 \%$ in larger series. ${ }^{1}$ The indication for such treatment remains controversial, as resection of a brainstem lesion itself carries significant risks. ${ }^{12}$ Early postoperative morbidity is $31 \%$, and long-term morbidity is around $18 \%$ according to the largest meta-analysis. ${ }^{10}$ As the natural course of a BSCM remains hard to predict, the indication for surgical treatment is highly individual and is balanced among the patient's present impairment due to the lesion, potential future hemorrhage events, and the estimated risks of surgical intervention. ${ }^{12}$ To guide these decisions, more detailed postoperative outcome data are needed; however, such data are scarce. In addition, data are oftentimes limited to mere descriptive clinical or functional outcome (disability) scores. Also, various and/or subjective outcome endpoints are utilized.10,13 Unfortunately, even functional scores may not completely reflect the patient's burden after treatment, particularly in those with minor functional impairment. ${ }^{14-16}$ This is especially so for brainstem lesions in which the interaction of brainstem nuclei, cranial nerves, and fiber tracts can cause very complex symptoms interfering with the patient's everyday activities even if they are not highly functionally disabling (balance problems, double vision, sensory disturbances, etc.). ${ }^{17}$ An optimal assessment of the patient's postoperative physical and psychological condition should therefore reflect such impairment, for example, by measuring health-related quality of life (HRQOL) ${ }^{18}$ anxiety and depression, ${ }^{19}$ or life satisfaction. ${ }^{20}$ Although these measures are not diseasespecific but are generic tools, they do provide information beyond mere ordinal functional scores and better reflect the patient's everyday life situation. So far, only a very few small studies have reported on, for example, postoperative HRQOL in BSCM. ${ }^{21-23}$ Because our center has a relatively high number of patients with BSCM undergoing surgery, we performed a cross-sectional outcome study including multiple patient- and physician-reported outcome surveys (functional outcome as measured by the modified Rankin Scale [mRS], HRQOL per the SF-36 instrument and the 9-item Life Satisfaction Questionnaire [LISAT-9], anxiety and depression as measured by the Hospital Anxiety and Depression Scale [HADS], patient-reported cranial nerve and brainstem symptoms, and return-to-work status). We also evaluated how these different surveys correlated with each other and with established relative and absolute clinical outcome endpoints after BSCM surgery. To put the results in context, we finally compared our results with the HRQOL and HADS scores in a healthy population.

\section{Methods}

\section{Study Design and Population}

We performed a cross-sectional study of all patients surgically treated for BSCM in our department between January 1, 2003, and December 31, 2019. A standardized interview was performed (baseline medical information, socio-educational background, SF-36 questionnaire, De- pression and Anxiety Subscales of the HADS [HADS-D and HADS-A, respectively], LISAT-9, and cranial nerve and brainstem symptoms questionnaire).

The study was conducted according to the principles expressed in the Declaration of Helsinki, and local ethics approval was obtained. Informed consent was obtained from all participants. The study was performed according to the STROBE protocol.

\section{Inclusion Criteria}

We included all patients aged $18-80$ years who had undergone surgical treatment for BSCM in the given time period with a minimum postoperative interval of 3 months and who agreed to participate.

\section{Exclusion Criteria}

We excluded patients who had undergone an invasive medical treatment necessitating hospitalization within 3 months prior to the interview and those with insufficient knowledge of the German language.

\section{Data Collection and Survey}

Clinical baseline data on patients and CCM were obtained based on medical charts according to CCM reporting standards ${ }^{6}$ both pre- and postoperatively (sex, age at surgery, CCM location in brainstem, history of multiple symptomatic hemorrhages, multiplicity of CCMs, known chronic disease [as defined by SF-36 criteria] ${ }^{18}$ known psychiatric disease, and degree of disability on the mRS). An experienced neuroradiologist independently assessed further radiological data (size of CCM, associated developmental venous anomaly [yes/no], and completeness of resection on postoperative MRI). In a standardized postoperative interview, we assessed HRQOL using the German version of the SF-36. The SF-36 questionnaire addresses 8 domains (physical functioning, role physical, bodily pain, general health perception, vitality, social functioning, role emotional, mental health, and the two component summary scores of physical health [PCS] and mental health [MCS]). Additionally, we assessed the German HADS score to evaluate symptom-based depression and anxiety (14-item questionnaire) and the LISAT-9 score to estimate satisfaction with life. The LISAT-9 contains 1 question about life satisfaction as a whole and 8 questions about domain-specific life satisfaction (selfcare ability, leisure time, vocational situation, financial situation, sexual life, partnership relations, family life, and contact with friends) and is scored on a 6-point scale ranging from very unsatisfied (1) to very satisfied (6). ${ }^{24}$ During the interview, we also queried the presence of any residual brainstem or cranial nerve symptoms (facial nerve palsy, hearing problems, ataxia, hemiparesis, balance disturbances, disturbance of fine motor skills, vision problems, double vision, nystagmus, deglutition disturbance, dysarthria, neuropathic pain, sensibility disturbance, and sexual dysfunction). The different symptoms were outlined in detail for each patient in a standardized way. We did not perform quantification of symptoms. Preoperatively, the employed patients were asked if and when they planned to return to their former work or equivalent 
positions. The interval between the questionnaire and surgery was calculated (months).

\section{Outcome Definitions \\ Relative Outcome}

A minimum 1-point increase in the mRS score compared to the preoperative score at the time of the interview was defined as neurological deterioration/operative morbidity.

\section{Absolute Outcome}

According to previous studies, ${ }^{25}$ an mRS score $\leq 2$ was defined as a favorable outcome, and an mRS score $>2$ was defined as an unfavorable outcome.

\section{Life Satisfaction Outcome}

According to previous studies, ${ }^{24}$ LISAT-9 scores of 1-4 were defined as unsatisfied and scores of 5-6 as satisfied.

\section{Reference Data}

HRQOL in the study population was compared to that in an age- and sex-matched healthy German population. ${ }^{18}$ HADS mean values in the study population were also compared to those in a healthy German population. ${ }^{19}$

\section{Statistical Analysis}

Statistical analysis was performed using IBM SPSS Statistics version 22 (IBM Corp.). Nominal data were expressed as absolute numbers and valid percent, and continuous variables were expressed as means \pm standard deviations. A Shapiro-Wilk test was used to test data for normal distribution. Additionally, histograms and Q-Q plots were used. We used parametric statistics for between-group comparisons. In comparing continuous variables, we used the unpaired t-test or Mann-Whitney U-test. For categorical variables, a chi-square or Fisher's exact test (expected frequencies < 5) was applied. Using a German reference sample, we created a sex- and age-matched 1:1 case control sample. A comparison of mean values (SF-36 scores) was performed using the Student $\mathrm{t}$-test preceded by Levene's test. Effect size was reported using Cohen's d.

To identify parameters with an impact on outcome/ scores, a bivariate correlation was performed. According to the variable character, Pearson's, Spearman's rho, or Kendall's tau-b test was used. Clinically relevant and significant parameters $(\mathrm{p}<0.05)$ were included in a linear or logistic regression analysis in terms of a stepwise model selection. To evaluate the association of outcome endpoints or functional scores with HRQOL scores, we performed a linear regression analysis and calculated $\mathrm{R}^{2}$ and the regression coefficient $\mathrm{B}$. To visualize the associations, we calculated receiver operating characteristic (ROC) curves and analyzed the areas under the curve (AUCs). All tests were two-tailed (alpha $=0.05)$.

\section{Results}

Seventy-four of 95 patients were eligible for inclusion in the study. Of the 21 patients excluded, 4 did not agree to participate and 17 did not have a complete data set or did not respond. The initial patient characteristics and post- operative functional outcome did not differ significantly between participating and nonparticipating patients (see Supplemental Table 1). Forty-five of the included patients $(61 \%)$ were female. The mean age was $40.4 \pm 12.10$ years (range 18-72 years). A chronic disease was found in 14 patients (19\%). A psychiatric disease was found in none of the patients. A history of symptomatic hemorrhage was found in all patients, and multiple hemorrhages were found in 37 patients $(50 \%)$. Eighteen patients (24\%) were initially unemployed or already retired; 56 patients (76\%) were employed. Table 1 shows further baseline patient and CCM characteristics.

\section{Outcome Endpoints}

Outcomes were measured at the last follow-up, which was at a mean of $53 \pm 44.5$ months (range 3-177 months). Fifteen patients (20\%) showed functional deterioration as indicated by a comparison to their preoperative $\mathrm{mRS}$ score (relative outcome endpoint). Sixty patients (81\%) showed a favorable outcome (mRS score $\leq 2$, absolute outcome endpoint). The mortality rate was $0 \%$ among the included patients. Thirty-eight (68\%) of the previously employed patients returned to their former positions; 18 (32\%) were unable to return to their previous employment. In 1 patient $(1 \%)$, resection of the CCM was rated as incomplete on postoperative MRI and revision surgery was performed. More details can be found in Table 1 and Fig. 1.

\section{Predictors of a Favorable Outcome}

Among the various parameters, only the size of the $\mathrm{CCM}$, initial mRS score $(\leq 2)$, and known chronic disease were significantly associated with a favorable outcome in the bivariate analysis. In the multivariate logistic regression analysis, only initial mRS score $(\leq 2)$ stayed significant $(\mathrm{p}=0.002$, OR $10.89,95 \%$ CI 2.36-50.25; Supplemental Table 2).

\section{SF-36 Results}

In the overall cohort, several SF-36 subdomains (physical functioning, role physical, general health perception, vitality, and social functioning) and the PCS showed significantly lower values compared to those in the healthy population. After stratification by outcome, patients with a favorable outcome showed results comparable to those in the healthy population, except for role physical (Cohen's d $=$ small effect) and PCS (Cohen's $\mathrm{d}=$ small effect). Patients with an unfavorable outcome showed decreased scores in all items except bodily pain, and the effects were mainly medium and large (Table 2). As expected, PCS scores tended to increase as the time from surgery increased, reflecting the typical neurological recovery from temporary deficits after surgery. Such an effect was not observed for MCS (Supplemental Fig. 1).

\section{HADS Results}

In the overall cohort, the mean HADS-A scores were $5.7 \pm 4.6$ for male patients and $5.1 \pm 3.3$ for female patients. The mean HADS-D scores were $5.1 \pm 4.7$ and $5.0 \pm 4.5$, respectively. Differences with the healthy population (as measured by effect size) were negligible except for slightly 
increased depression symptoms in females. After stratification by outcome, patients with a favorable outcome showed normal values. Patients with an unfavorable outcome showed significantly increased anxiety and depression symptoms (male) or exclusively increased depression symptoms (female), all with a medium effect size (Table 2).

\section{LISAT-9 Results}

Results of the LISAT-9 are listed in Table 3. Patients with a favorable outcome showed significantly better results than the patients with an unfavorable outcome (except for partnership and family life). To put the results in context, we added "reference scores" for a healthy population sample ${ }^{30}$ and a sample of patients 1 year after stroke. ${ }^{31}$

\section{Patient-Reported Cranial Nerve and Brainstem Symptoms}

Details of frequencies in patient-reported symptoms are listed in Table 4. Bivariate analysis showed significant associations for several symptoms, especially physical HRQOL (PCS). In the multivariate analysis, none of the associations remained significant. In a further sensitivity analysis, we assessed different combinations of symptoms (both being present in one patient). Here we found that especially balance disturbance (balance disorder and ataxia) was significantly associated with physical HRQOL (multivariate, $\mathrm{p}=$ 0.000 , coefficient $=-11.459$; Supplemental Table 3).

\section{Correlation of HRQOL With Established Outcome Endpoints}

Postoperative mRS strongly correlated with PCS ( $\mathrm{p}=$ $\left.0.000, R^{2}=0.49\right)$ and less strongly with MCS $(p=0.001$, $\left.\mathrm{R}^{2}=0.14\right)$. However, the variance of mean PCS values significantly increased with the mRS score: from 4.2 (mRS 0) to 68.3 (mRS 1) to 98.8 (mRS 2) and to 118.7 (mRS 3).

Absolute outcome endpoint ( $\mathrm{mRS} \leq 2)$ strongly correlated with PCS $\left(\mathrm{p}=0.000, \mathrm{R}^{2}=0.28\right)$ and less with MCS $\left(p=0.002, R^{2}=0.12\right)$. These values were lower/not significant for the relative outcome endpoint (deterioration of at least 1 point in the mRS score; $p=0.003, R^{2}=0.12$ and $p$ $=0.191, R^{2}=0.02$, respectively). Accordingly, AUC values in the ROC analysis showed good and fair values for the absolute outcome endpoint for PCS and MCS, respectively (AUC $=0.870$ and 0.743). The relative outcome endpoint showed only fair and poor accuracy $(\mathrm{AUC}=0.729$ and 0.620; Fig. 2).

\section{Discussion}

We present a detailed outcome analysis of a large cohort of patients after surgery for BSCM. According to the most recent meta-analysis, ${ }^{10}$ our series is the sixth largest single-center series of those published since 1986. The meta-analysis summarized 86 studies comprising 2493 patients (range 3-397 patients, median 11.5 per series), demonstrating how rarely surgery for BSCM is performed. Most series, however, have reported outcome solely based on functional scores or clinical evaluation. Further outcome measurements, including HRQOL, have only been reported in 3 other series with $17,2222,,^{23}$ and $71^{21}$ patients.

Compared to meta-analysis results, ${ }^{9,10}$ the long-term
TABLE 1. Baseline characteristics and outcome in 74 patients with BSCM treated with surgery

\begin{tabular}{|c|c|}
\hline Variable & Value \\
\hline \multicolumn{2}{|l|}{ Baseline characteristics $(n=74)$} \\
\hline Female sex & $45(61 \%)$ \\
\hline Mean age in yrs & $40.4 \pm 12.10$ \\
\hline Chronic disease & $14(19 \%)$ \\
\hline Psychiatric disease & $0(0 \%)$ \\
\hline Multiple CCMs & $5(7 \%)$ \\
\hline Multiple SHs & $37(50 \%)$ \\
\hline \multicolumn{2}{|l|}{ Initial mRS score } \\
\hline 0 & $2(3 \%)$ \\
\hline 1 & $26(35 \%)$ \\
\hline 2 & $24(32 \%)$ \\
\hline 3 & $13(18 \%)$ \\
\hline 4 & $9(12 \%)$ \\
\hline 5 & $0(0 \%)$ \\
\hline Mean BSCM size in $\mathrm{mm}$ & $17.8 \pm 7.3$ \\
\hline Associated DVA & $22(30 \%)$ \\
\hline \multicolumn{2}{|l|}{ Location } \\
\hline Medullary & $7(9 \%)$ \\
\hline Pontomedullary & $15(20 \%)$ \\
\hline Pontine & $30(41 \%)$ \\
\hline Pontomesenphalic & $8(11 \%)$ \\
\hline Mesencephalic & $14(19 \%)$ \\
\hline \multicolumn{2}{|l|}{ Ouctome $(n=74)$} \\
\hline Mean time since surgery in mos (range) & $53 \pm 44.5(3-177)$ \\
\hline Functional deterioration at last FU & $15(20 \%)$ \\
\hline Functional score equal or better at last FU & $59(80 \%)$ \\
\hline Favorable outcome & $60(81 \%)$ \\
\hline Unfavorable outcome & $14(19 \%)$ \\
\hline Mortality & $0(0 \%)$ \\
\hline \multicolumn{2}{|l|}{ mRS score at last FU } \\
\hline 0 & $8(11 \%)$ \\
\hline 1 & $27(37 \%)$ \\
\hline 2 & $25(34 \%)$ \\
\hline 3 & $7(10 \%)$ \\
\hline 4 & $6(8 \%)$ \\
\hline 5 & $1(1 \%)$ \\
\hline RTW & $38\left(68 \%{ }^{*}, 76 \% \dagger\right)$ \\
\hline Mean time to RTW in mos (range) & $7.6 \pm 5.7(1-24)$ \\
\hline \multicolumn{2}{|l|}{$\begin{array}{l}\text { Favorable outcome as stratified by initial } \\
\text { mRS score }\end{array}$} \\
\hline 0 & $2(100 \%)$ \\
\hline 1 & $26(100 \%)$ \\
\hline 2 & $21(88 \%)$ \\
\hline 3 & $5(38 \%)$ \\
\hline 4 & $6(67 \%)$ \\
\hline
\end{tabular}

DVA = deep venous anomaly; FU = follow-up; $n=$ number of patients; $R T W=$ return to work; $\mathrm{SH}=$ symptomatic hemorrhage.

Values are expressed as number (\%) of patients or mean \pm standard deviation.

* Among the previously employed.

$\dagger$ Among those with a favorable outcome who were previously employed. 

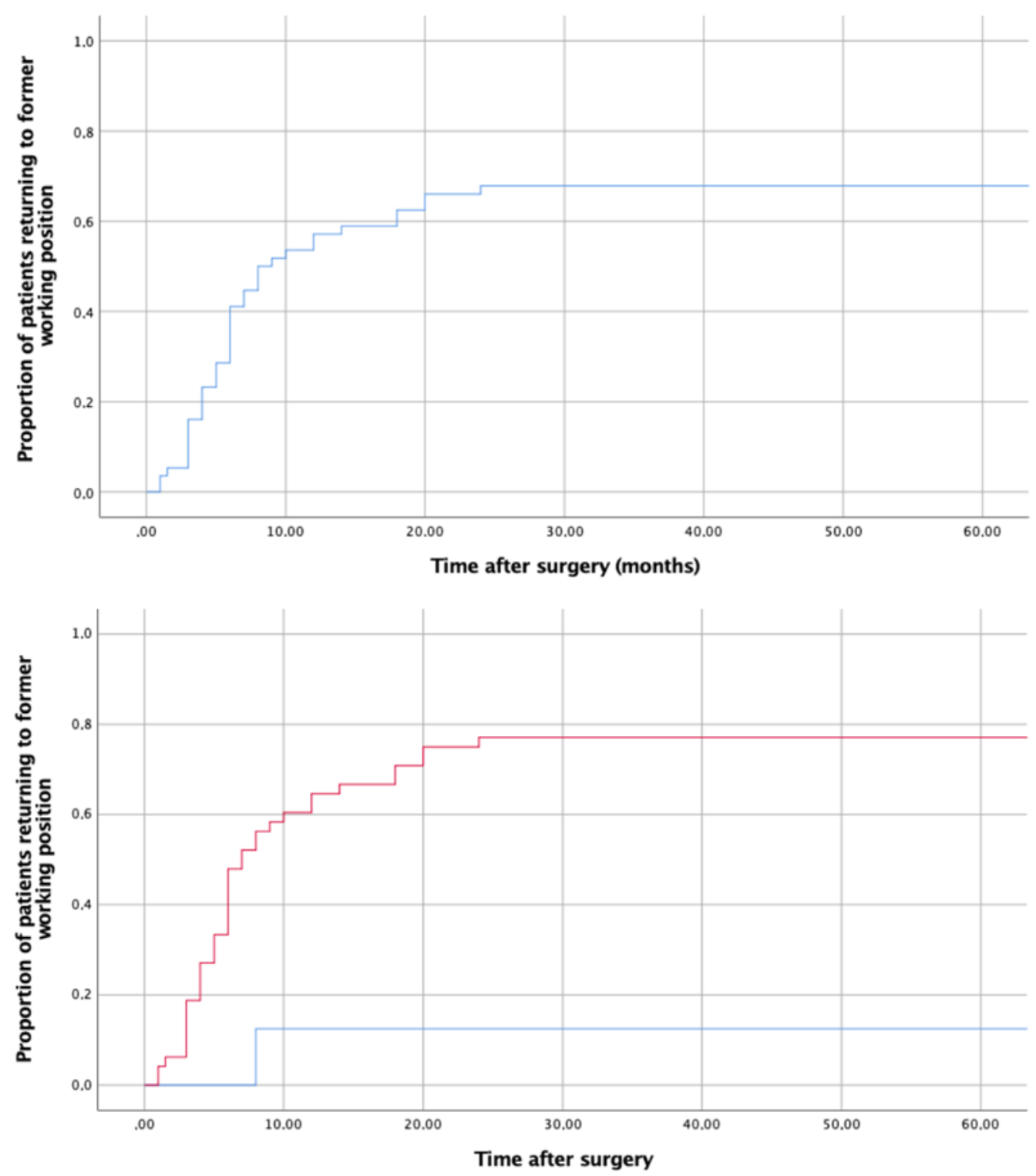

FIG. 1. Proportion of patients returning to former employment after surgery. Upper: Overall cohort. Lower: Patients stratified by outcome. $R e d=$ favorable outcome; blue = unfavorable outcome. Figure is available in color online only.

relative functional outcome in our series (20\% functional deterioration) was within the same range $(18.3 \%$ and $16 \%$, respectively). Compared to the largest single-center series defining and reporting absolute functional scorebased outcomes, our series showed identical results $(19 \%$ vs $20.3 \%$, respectively unfavorable outcome [mRS $>2]$ ). ${ }^{25}$ The mean age and distribution of CCM over the brainstem were also comparable to those in other large series..$^{25,26}$ Overall, our study lies within the range of functional outcomes and patient characteristics reported in other series, which increases the external validity of our additionally reported outcomes.

The overall cohort showed significantly impaired HRQOL subdomains and component scores compared to those in an age- and sex-matched healthy population. Scores were strongly decreased in patients with an unfavorable outcome. However, in patients with a favorable outcome, the PCS was slightly decreased compared to that in the healthy population. Notably, a decrease of 2 points in the component scores (and 5 points in the subdomains) is regarded as a clinically and psychosocially relevant decrease. ${ }^{27,28}$

The impact on patients with an unfavorable outcome is also reflected by the strongly increased depression and anxiety scores compared to those in the healthy population. Also, the relatively low rate of patients returning to their former employment reflects this (approximately 80\% with a favorable outcome, approximately $10 \%$ with an unfavorable outcome). For example, after unruptured aneurysm treatment, this rate is around $90 \% .^{29}$

While satisfaction in life has not been previously assessed in BSCM and healthy population data are limited, the results are difficult to put into context. Compared with 
TABLE 2. SF-36 and HADS-A and -D results for the entire cohort and subgroups

\begin{tabular}{|c|c|c|c|c|c|c|c|c|c|c|c|c|c|c|}
\hline \multirow[b]{2}{*}{ Scale } & \multicolumn{4}{|c|}{ Overall Cohort $(n=74)$} & \multicolumn{4}{|c|}{ Favorable Outcome $(n=60)$} & \multicolumn{4}{|c|}{ Unfavorable Outcome $(n=14)$} & \multicolumn{2}{|c|}{$\begin{array}{c}\text { Reference } \\
\text { Sample* }(n=74)\end{array}$} \\
\hline & Mean & SD & $\mathrm{p}$ Value $\dagger$ & $\begin{array}{l}\text { Effect } \\
\text { Sizef }\end{array}$ & Mean & SD & $\mathrm{p}$ Value $†$ & $\begin{array}{l}\text { Effect } \\
\text { Size }\end{array}$ & Mean & SD & $\mathrm{p}$ Value $\dagger$ & $\begin{array}{l}\text { Effect } \\
\text { Sizeł }\end{array}$ & Mean & SD \\
\hline \multicolumn{15}{|c|}{ SF-36 scores } \\
\hline \multicolumn{15}{|c|}{$\begin{array}{l}\text { Physical } \\
\text { health } \\
\text { subdomains }\end{array}$} \\
\hline PF & 68.37 & 32.90 & 0.000 & Small & 78.66 & 24.40 & 0.080 & NA & 24.28 & 28.20 & 0.000 & Large & 86.01 & 23.43 \\
\hline $\mathrm{RP}$ & 53.26 & 45.53 & 0.000 & Small & 62.36 & 42.78 & 0.003 & Small & 14.28 & 36.31 & 0.000 & Medium & 82.77 & 33.59 \\
\hline $\mathrm{BP}$ & 80.47 & 27.62 & 0.578 & NA & 83.31 & 25.39 & 0.248 & NA & 68.28 & 34.08 & 0.266 & NA & 77.89 & 28.47 \\
\hline $\mathrm{GH}$ & 61.97 & 23.48 & 0.003 & Small & 66.82 & 20.43 & 0.111 & NA & 41.21 & 25.09 & 0.000 & Medium & 72.12 & 17.02 \\
\hline VT & 52.56 & 20.24 & 0.024 & Small & 56.16 & 19.29 & 0.291 & NA & 37.14 & 17.17 & 0.000 & Medium & 59.52 & 16.80 \\
\hline \multicolumn{15}{|c|}{$\begin{array}{l}\text { Mental health } \\
\text { subdomains }\end{array}$} \\
\hline SF & 71.79 & 31.31 & 0.001 & Small & 80.00 & 26.85 & 0.173 & NA & 36.60 & 24.25 & 0.000 & Medium & 85.64 & 19.03 \\
\hline RE & 71.62 & 41.17 & 0.125 & NA & 78.88 & 37.31 & 0.704 & NA & 40.47 & 43.71 & 0.000 & Medium & 81.27 & 34.24 \\
\hline $\mathrm{MH}$ & 69.83 & 17.93 & 0.892 & NA & 73.46 & 16.20 & 0.247 & NA & 54.28 & 17.16 & 0.001 & Medium & 70.21 & 15.90 \\
\hline \multicolumn{15}{|c|}{$\begin{array}{l}\text { Component } \\
\text { scores }\end{array}$} \\
\hline PCS & 44.61 & 11.71 & 0.000 & Small & 47.59 & 9.98 & 0.047 & Small & 31.80 & 10.02 & 0.000 & Medium & 51.20 & 10.55 \\
\hline MCS & 47.51 & 10.82 & 0.537 & NA & 49.32 & 9.82 & 0.643 & NA & 39.78 & 11.84 & 0.003 & Small & 47.51 & 9.28 \\
\hline \multicolumn{15}{|c|}{ HADS scores } \\
\hline \multicolumn{15}{|c|}{ HADS-A } \\
\hline M & 5.7 & 4.6 & 0.253 & NA & 4.9 & 3.5 & 0.682 & NA & 9.1 & 3.9 & 0.0001 & Medium & 5.1 & 3.0 \\
\hline F & 5.1 & 3.3 & 0.011 & None & 4.9 & 4.5 & 0.019 & None & 6.0 & 2.2 & 0.732 & NA & 6.3 & 3.2 \\
\hline \multicolumn{15}{|l|}{ HADS-D } \\
\hline M & 5.1 & 4.7 & 0.014 & None & 3.7 & 3.4 & 0.99 & NA & 10.3 & 4.3 & 0.0001 & Medium & $3.7 \S$ & $2.7 \S$ \\
\hline $\mathrm{F}$ & 5.0 & 4.5 & 0.001 & Small & 3.7 & 3.8 & 0.322 & NA & 10.6 & 4.8 & 0.0001 & Medium & $3.2 \S$ & $2.6 \S$ \\
\hline
\end{tabular}

$\mathrm{BP}=$ bodily pain; $\mathrm{GH}=$ general health perception; $\mathrm{MH}=$ mental health; $\mathrm{NA}=$ not available; $\mathrm{PF}=$ physical functioning; $\mathrm{RE}=$ role emotional; $\mathrm{RP}=$ role physical; $\mathrm{SF}=$ social functioning; $\mathrm{VT}=$ vitality.

Boldface type indicates statistical significance.

${ }^{*}$ Age- and sex-matched sample from German reference cohort.

$\dagger$ As compared to age- and sex-matched reference sample (Student t-test).

$\ddagger$ As measured by Cohen's d: >0.2, small effect; $>0.5$, medium effect; $>0.8$, large effect.

$\S$ Reference values for HADS-D are normative values from the German population.

TABLE 3. LISAT-9 results

\begin{tabular}{|c|c|c|c|c|c|c|}
\hline Domain & $\begin{array}{l}\text { Overall Cohort } \\
\qquad(n=63)\end{array}$ & $\begin{array}{l}\text { Favorable Outcome } \\
\qquad(\mathrm{n}=52)\end{array}$ & $\begin{array}{l}\text { Unfavorable Outcome } \\
\qquad(n=11)\end{array}$ & p Value* & $\begin{array}{l}\text { Nonspecific Patient } \\
\text { Population }(n=69) \dagger\end{array}$ & $\begin{array}{c}\text { Patients } 1 \text { Year After } \\
\text { Stroke }(n=119) \ddagger\end{array}$ \\
\hline Life as a whole & $31(49 \%)$ & $31(60 \%)$ & $0(0 \%)$ & 0.000 & $75 \%$ & $49 \%$ \\
\hline Self-care ability & $40(63 \%)$ & $37(71 \%)$ & $3(27 \%)$ & 0.006 & $100 \%$ & $90 \%$ \\
\hline Leisure time & $30(48 \%)$ & $29(56 \%)$ & $1(9 \%)$ & 0.005 & $71 \%$ & $48 \%$ \\
\hline Vocational situation & $27(43 \%)$ & $26(50 \%)$ & $1(9 \%)$ & 0.013 & $69 \%$ & $55 \%$ \\
\hline Financial situation & $36(57 \%)$ & $33(63 \%)$ & $3(27 \%)$ & 0.028 & $48 \%$ & $59 \%$ \\
\hline Sexual life & $30(48 \%)$ & $29(56 \%)$ & $1(9 \%)$ & 0.005 & $65 \%$ & $33 \%$ \\
\hline Partnership & $46(73 \%)$ & $40(77 \%)$ & $6(55 \%)$ & 0.129 & $64 \%$ & $72 \%$ \\
\hline Family life & $51(81 \%)$ & $44(85 \%)$ & $7(64 \%)$ & 0.107 & $74 \%$ & $78 \%$ \\
\hline Contact w/ friends & $47(75 \%)$ & $44(85 \%)$ & $3(27 \%)$ & 0.000 & $81 \%$ & $58 \%$ \\
\hline
\end{tabular}

Values are expressed as number (\%), representing the satisfied patients. LISAT-9 scores of 5-6 were defined as satisfied. Boldface type indicates statistical signifi-

cance.

* Favorable and unfavorable outcomes compared using the chi-square test.

$\dagger$ Patients reporting satisfaction before trauma surgery (normal population).

$\ddagger$ Patients reporting 1 year after a stroke. 
TABLE 4. Patient-reported cranial nerve and brainstem symptoms

\begin{tabular}{|c|c|c|c|c|c|c|c|c|c|c|}
\hline \multirow[b]{2}{*}{ Symptom } & \multirow{2}{*}{$\begin{array}{c}\text { Frequency } \\
\text { Among } \\
\text { Overall } \\
\text { Cohort } \\
(n=74)\end{array}$} & \multirow{2}{*}{$\begin{array}{c}\text { Frequency } \\
\text { Among } \\
\text { Favorable } \\
\text { Outcome } \\
(n=60)\end{array}$} & \multicolumn{2}{|c|}{$\begin{array}{c}\text { Association w/ } \\
\text { Impairment of } \\
\text { Physical HRQOL* } \\
\text { (bivariate) }\end{array}$} & \multicolumn{2}{|c|}{$\begin{array}{l}\text { Association w/ } \\
\text { Impairment of } \\
\text { Physical HRQOL* } \\
\text { (multivariate) }\end{array}$} & \multicolumn{2}{|c|}{$\begin{array}{c}\text { Association w/ } \\
\text { Impairment of } \\
\text { Mental HRQOL† } \\
\text { (bivariate) }\end{array}$} & \multicolumn{2}{|c|}{$\begin{array}{l}\text { Association w/ } \\
\text { Impairment of } \\
\text { Mental HRQOL } † \\
\text { (multivariate) }\end{array}$} \\
\hline & & & Coefficient & $p$ Value & Coefficient & $p$ Value & Coefficient & $p$ Value & Coefficient & $\mathrm{p}$ Value \\
\hline \multicolumn{11}{|l|}{ Facial nerve palsy-dysarthria } \\
\hline Facial nerve palsy & $21(28 \%)$ & $6(10 \%)$ & -0.196 & 0.043 & -3.688 & 0.177 & -0.058 & 0.550 & NA & NA \\
\hline Dysarthria & $14(19 \%)$ & $8(13 \%)$ & -0.298 & 0.001 & 0.027 & 0.994 & -0.132 & 0.172 & NA & NA \\
\hline Hearing impairment & $23(31 \%)$ & $18(30 \%)$ & -0.061 & 0.529 & NA & NA & -0.124 & 0.200 & NA & NA \\
\hline \multicolumn{11}{|l|}{ Motor symptoms } \\
\hline Hemiparesis & $18(24 \%)$ & $10(17 \%)$ & -0.338 & 0.000 & -4.885 & 0.087 & -0.033 & 0.734 & NA & NA \\
\hline Fine motor skills impairment & $45(61 \%)$ & $31(52 \%)$ & -0.301 & 0.002 & -0.272 & 0.919 & -0.235 & 0.015 & -2.434 & 0.403 \\
\hline \multicolumn{11}{|l|}{ Balance } \\
\hline Balance disorder & $44(59 \%)$ & $31(52 \%)$ & 0.364 & 0.000 & -3.864 & 0.141 & -0.194 & 0.045 & -2.072 & 0.449 \\
\hline Ataxia & $35(47 \%)$ & $21(35 \%)$ & 0.444 & 0.000 & -5.351 & 0.071 & -0.265 & 0.006 & -2.241 & 0.451 \\
\hline \multicolumn{11}{|l|}{ Vision impairment } \\
\hline Vision impairment & $23(31 \%)$ & $12(20 \%)$ & -0.239 & 0.014 & -0.246 & 0.935 & -0.277 & 0.004 & -4.380 & 0.127 \\
\hline Double vision & $30(41 \%)$ & $21(35 \%)$ & -0.273 & 0.005 & -1.622 & 0.548 & -0.075 & 0.439 & NA & NA \\
\hline Nystagmus & $13(18 \%)$ & $7(12 \%)$ & -0.328 & 0.001 & -5.654 & 0.133 & -0.179 & 0.065 & NA & NA \\
\hline Surgery for double vision & $9(12 \%)$ & $8(13 \%)$ & & & & & & & & \\
\hline Caudal cranial nerve impairment & $7(9 \%)$ & $5(8 \%)$ & -0.005 & 0.963 & NA & NA & -0.021 & 0.829 & NA & NA \\
\hline \multicolumn{11}{|l|}{ Sensory disorder } \\
\hline Sensory disorder & $38(51 \%)$ & $29(48 \%)$ & -0.220 & 0.023 & -2.451 & 0.329 & 0.021 & 0.825 & NA & NA \\
\hline Pain & $19(26 \%)$ & $14(23 \%)$ & -0.305 & 0.002 & -1.050 & 0.707 & -0.194 & 0.045 & -3.211 & 0.127 \\
\hline Sexual dysfunction & $14(19 \%)$ & $8(13 \%)$ & -0.333 & 0.001 & -2.458 & 0.476 & -0.086 & 0.377 & NA & NA \\
\hline
\end{tabular}

Values expressed as number (\%), representing satisfied patients. Boldface type indicates statistical significance.

${ }^{*}$ Physical component score used.

$\dagger$ Mental component score used.

that in unaffected patients ${ }^{30}$ or patients 1 year after stroke, ${ }^{31}$ it is notable that especially satisfaction in self-care ability was low, even in cases with a favorable outcome. An unfavorable outcome led to a massive reduction in satisfaction in all domains of life (except family life and partnership).

Cranial nerve and brainstem symptoms in this series were assessed using patient-reported categorical data. This may have led to an overreporting of symptoms (whereas physician-reported outcomes are prone to underreporting of symptoms). ${ }^{32}$ However, it has been shown that patientreported symptoms enhance HRQOL assessment and outcome discrimination in clinical trials. ${ }^{32}$ Most importantly, we found a strong association between balance disorders and HRQOL, meaning that among cranial nerve and brainstem symptoms, any disturbance in balance and the presence of ataxia reduced physical (and less mental) HRQOL most significantly. Additionally, to the best of our knowledge, sexual dysfunction (and satisfaction with sexual life), a potential postoperative complication and important aspect of HRQOL, has been assessed for the first time with this study.

Regarding outcome reporting after BSCM surgery in general, an important finding of our study was that HRQOL is more precisely reflected by using absolute outcome endpoints, that is, based on the mRS, rather than relative outcome endpoints. The general superiority of absolute over relative outcome measures in evaluating outcome has been discussed elsewhere. ${ }^{33}$ To note, variance in the HRQOL component scores increased significantly as the $\mathrm{mRS}$ score increased. This means that in individual cases, lower mRS outcome scores of 1 or 2 were also associated with decreased HRQOL and vice versa in this study.

In summary, this study meticulously reflects the potential multidimensional impact on patients after surgery for BSCM. To note, nearly all patients with an unfavorable outcome were already initially severely disabled (mRS > 2) by the CCM hemorrhage itself. This is, in general, not infrequent after BSCM hemorrhage. ${ }^{8}$ Only $6 \%$ of patients with a preoperative $\mathrm{mRS}$ score of $0-2$ experienced an unfavorable outcome in this series. Accordingly, a preoperative mRS score of 0-2 was an independent predictor of a favorable outcome ( $\mathrm{p}=0.002$, OR $10.89,95 \%$ CI 2.36-50.25). However, the natural course even after a severe bleeding, as well as the potential recovery from symptoms, remains hard to predict and could be benign. ${ }^{11}$ In addition, even patients with favorable outcomes frequently reported residual symptoms, especially those that were strongly associated with a decreased HRQOL (52\% balance disorders). A satisfaction rate of only $71 \%$ in self-care ability also reflects substantial impairment in everyday life in these patients.

Although patients may live a relatively "normal" life after resection of their BSCM and attain a favorable outcome, the burden after surgery is substantial when this is not achieved. This dilemma emphasizes the importance 

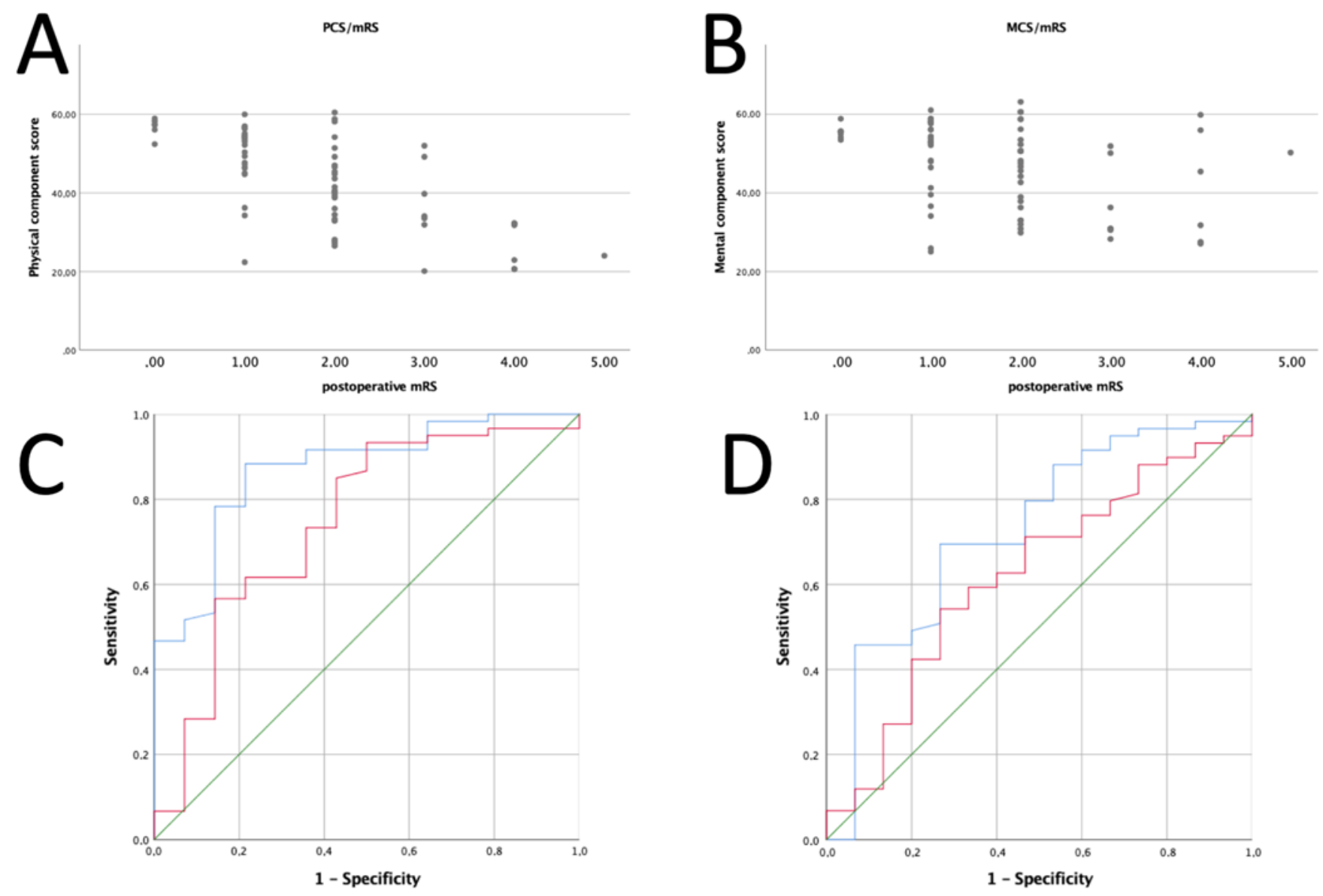

FIG. 2. Correlation of functional outcome and SF-36 scores. Scatter plots for PCS (A) and MCS (B) stratified by postoperative $\mathrm{mRS}$ score. Especially the PCS shows high variance at mRS scores 1 and 2. AUCs show the correlation of outcome endpoints with PCS (C) and MCS (D). Notably, the absolute outcome measurement (blue) correlates better compared to the relative outcome measurement $(r e d)$. Figure is available in color online only.

in further improving the identification of "ideal" candidates for surgery and specifying these indications in future guidelines. Moreover, we should be aware that a "favorable outcome" after BSCM surgery as we define it does not necessarily reflect a good HRQOL or satisfaction in life in all patients (especially in those with an mRS score of 2). This is even more relevant when using relative outcome endpoints.

\section{Study Limitations}

This study has several limitations. It is not a comparative study with randomized treatment allocation. The presumed natural untreated course of patients remains unclear, and outcome cannot be compared to that in untreated or otherwise-treated BSCM. The initial selection for treatment was not based on predefined criteria, as such criteria do not exist yet.

Some data were assessed retrospectively and are thus susceptible to information and selection bias. Although this is a relatively large series, absolute numbers were small, probably over- or underestimating effects. Longitudinal data offer more information, but they were not available in our study. In addition, preoperative scores after di- agnosis of symptomatic hemorrhage of a BSCM would be difficult to put into context, as these acute situations interfere with HRQOL perception. ${ }^{18}$ Comparisons with healthy populations (as performed in this study) may deliver even more reliable results.

\section{Conclusions}

Our data can improve patient counseling and decisionmaking in BSCM treatment and may function as a benchmark. We report outcomes after BSCM surgery in high detail, emphasizing the impact of cranial nerve and brainstem symptoms on HRQOL. When reporting BSCM surgery outcomes, absolute outcome endpoints should be applied.

\section{References}

1. Horne MA, Flemming KD, Su IC, et al. Clinical course of untreated cerebral cavernous malformations: a meta-analysis of individual patient data. Lancet Neurol. 2016;15(2):166-173.

2. Al-Shahi Salman R, Hall JM, Horne MA, et al. Untreated clinical course of cerebral cavernous malformations: a prospective, population-based cohort study. Lancet Neurol. 2012; 11(3):217-224. 
3. Labauge $\mathrm{P}$, Brunereau L, Lévy $\mathrm{C}$, et al. The natural history of familial cerebral cavernomas: a retrospective MRI study of 40 patients. Neuroradiology. 2000;42(5):327-332.

4. Rigamonti D, Drayer BP, Johnson PC, et al. The MRI appearance of cavernous malformations (angiomas). J Neurosurg. 1987;67(4):518-524

5. Zabramski JM, Wascher TM, Spetzler RF, et al. The natural history of familial cavernous malformations: results of an ongoing study. J Neurosurg. 1994;80(3):422-432.

6. Al-Shahi Salman R, Berg MJ, Morrison L, Awad IA. Hemorrhage from cavernous malformations of the brain: definition and reporting standards. Stroke. 2008;39(12):3222-3230.

7. Flemming KD, Link MJ, Christianson TJ, Brown RD Jr. Prospective hemorrhage risk of intracerebral cavernous malformations. Neurology. 2012;78(9):632-636.

8. Dammann P, Jabbarli R, Wittek P, et al. Solitary sporadic cerebral cavernous malformations: risk factors of first or recurrent symptomatic hemorrhage and associated functional impairment. World Neurosurg. 2016;91:73-80.

9. Gross BA, Batjer HH, Awad IA, et al. Brainstem cavernous malformations: 1390 surgical cases from the literature. World Neurosurg. 2013;80(1-2):89-93.

10. Kearns KN, Chen CJ, Tvrdik P, et al. Outcomes of surgery for brainstem cavernous malformations: a systematic review. Stroke. 2019;50(10):2964-2966.

11. Li D, Wu ZY, Liu PP, et al. Natural history of brainstem cavernous malformations: prospective hemorrhage rate and adverse factors in a consecutive prospective cohort. J Neurosurg. Published online March 13, 2020. doi:10.3171/2019.12. JNS192856

12. Akers A, Al-Shahi Salman R, Awad IA, et al. Synopsis of guidelines for the clinical management of cerebral cavernous malformations: consensus recommendations based on systematic literature review by the Angioma Alliance Scientific Advisory Board Clinical Experts Panel. Neurosurgery. 2017; 80(5):665-680.

13. Poorthuis M, Samarasekera N, Kontoh K, et al. Comparative studies of the diagnosis and treatment of cerebral cavernous malformations in adults: systematic review. Acta Neurochir (Wien). 2013;155(4):643-649.

14. Weimar C, Kurth T, Kraywinkel K, et al. Assessment of functioning and disability after ischemic stroke. Stroke. 2002;33(8):2053-2059.

15. Contopoulos-Ioannidis DG, Karvouni A, Kouri I, Ioannidis JP. Reporting and interpretation of SF-36 outcomes in randomised trials: systematic review. BMJ. 2009;338:a3006.

16. Ali M, Fulton R, Quinn T, Brady M. How well do standard stroke outcome measures reflect quality of life? A retrospective analysis of clinical trial data. Stroke. 2013;44(11):3161-3165.

17. Chua KS, Kong KH. Functional outcome in brain stem stroke patients after rehabilitation. Arch Phys Med Rehabil. 1996; 77(2):194-197.

18. Bullinger M. German translation and psychometric testing of the SF-36 Health Survey: preliminary results from the IQOLA Project. International Quality of Life Assessment. Soc Sci Med. 1995;41(10):1359-1366.

19. Hinz A, Brähler E. Normative values for the Hospital Anxiety and Depression Scale (HADS) in the general German population. J Psychosom Res. 2011;71(2):74-78.

20. Röding J, Glader EL, Malm J, Lindström B. Life satisfaction in younger individuals after stroke: different predisposing factors among men and women. J Rehabil Med. 2010;42(2):155-161.

21. Dukatz T, Sarnthein J, Sitter H, et al. Quality of life after brainstem cavernoma surgery in 71 patients. Neurosurgery. 2011;69(3):689-695.

22. Cornelius JF, Kürten K, Fischer I, et al. Quality of life after surgery for cerebral cavernoma: brainstem versus nonbrainstem location. World Neurosurg. 2016;95:315-321.

23. Lashkarivand A, Ringstad G, Eide PK. Surgery for brainstem cavernous malformations: association between preoperative grade and postoperative quality of life. Oper Neurosurg (Hagerstown). 2020;18(6):590-598.

24. Backes D, Rinkel GJ, van der Schaaf IC, et al. Recovery to preinterventional functioning, return-to-work, and life satisfaction after treatment of unruptured aneurysms. Stroke. 2015;46(6):1607-1612.

25. Garcia RM, Ivan ME, Lawton MT. Brainstem cavernous malformations: surgical results in 104 patients and a proposed grading system to predict neurological outcomes. Neurosurgery. 2015;76(3):265-278.

26. Abla AA, Lekovic GP, Turner JD, et al. Advances in the treatment and outcome of brainstem cavernous malformation surgery: a single-center case series of 300 surgically treated patients. Neurosurgery. 2011;68(2):403-415.

27. Ware JE Jr, Kosinski M, Bayliss MS, et al. Comparison of methods for the scoring and statistical analysis of SF-36 health profile and summary measures: summary of results from the Medical Outcomes Study. Med Care. 1995;33(4) (suppl):AS264-AS279.

28. McHorney CA, Ware JE Jr. Construction and validation of an alternate form general mental health scale for the Medical Outcomes Study Short-Form 36-Item Health Survey. Med Care. 1995;33(1):15-28.

29. Dammann P, Wittek P, Darkwah Oppong M, et al. Relative health-related quality of life after treatment of unruptured intracranial aneurysms: long-term outcomes and influencing factors. Ther Adv Neurol Disord. 2019;12:1756286419833492.

30. Anke AG, Fugl-Meyer AR. Life satisfaction several years after severe multiple trauma-a retrospective investigation. Clin Rehabil. 2003;17(4):431-442.

31. Visser-Meily A, Post M, van de Port I, et al. Psychosocial functioning of spouses in the chronic phase after stroke: improvement or deterioration between 1 and 3 years after stroke? Patient Educ Couns. 2008;73(1):153-158.

32. Deshpande PR, Rajan S, Sudeepthi BL, Abdul Nazir CP. Patient-reported outcomes: a new era in clinical research. Perspect Clin Res. 2011;2(4):137-144.

33. Stegenga J. Measuring effectiveness. Stud Hist Philos Biol Biomed Sci. 2015;54:62-71.

\section{Disclosures}

The authors report no conflict of interest concerning the materials or methods used in this study or the findings specified in this paper.

\section{Author Contributions}

Conception and design: Dammann, Sure. Acquisition of data: Dammann, Herten, Chen, Forsting, Kleinschnitz. Analysis and interpretation of data: Dammann, Forsting, Kleinschnitz. Drafting the article: Dammann. Critically revising the article: Dammann, Santos, Kleinschnitz, Sure. Reviewed submitted version of manuscript: Herten, Santos, Rauschenbach, Chen, Darkwah Oppong, Sure. Approved the final version of the manuscript on behalf of all authors: Dammann. Statistical analysis: Schmidt. Administrative/technical/material support: Darkwah Oppong, Schmidt.

\section{Supplemental Information \\ Online-Only Content}

Supplemental material is available with the online version of the article.

Supplemental Tables and Figure. https://thejns.org/doi/suppl/ 10.3171/2020.6.JNS201823.

\section{Correspondence}

Philipp Dammann: University Hospital Essen, Germany. philipp.dammann@uk-essen.de. 